\title{
Chondrodystrophic dwarfism and multiple malformations in two sisters
}

\author{
U.E. Pazzaglia ${ }^{1}$, L.Pedrotti ${ }^{1}$, G. Beluffi ${ }^{2}$ and L. Ceciliani ${ }^{1}$ \\ ${ }^{1}$ Clinica Ortopedica dell'Università di Pavia, and \\ ${ }^{2}$ Servizio di Radiodiagnostica, Sezione di Radiopediatria, I. R.C.C.S. Policlinico San Matteo, Pavia, Italy
}

\begin{abstract}
A genetic skeletal dysplasia with dwarfism, scoliosis and multiple skeletal defects was observed in two sisters. Only nine cases with similar features have been reported in the literature.
\end{abstract}

A syndrome presenting with dwarfism, spondylometaphyseal abnormalities and advanced bone age was reported by Neimann and colleagues in 1964 [1] and by Desbuquois and colleagues in 1966 [2]. Other cases with similar features were described by Piussan and colleagues [3], who distinguished this condition from Larsen's Syndrome.

We report two new observations of this rare disease.

\section{Case reports}

Case 1

Was the first daughter of consanguineous parents. The father had a short stature, but no cases of dwarfism were observed in the family pedigree.

The pregnancy and the delivery were uneventful; a mild bilateral club foot was observed at birth and it was treated by simple manipulation.

We first observed the child at the age of three years because of bilateral hip dislocation and scoliosis.

The head was abnormally large with protrusion of the frontal bones; the fontanels were closed; the face showed protruding eyes, saddle nose and hypertrophy of the upper jaw and zygomatic bones. The mandible, on the contrary, was small and the teeth were normal. The neck was short. The trunk was deformed by scoliosis. Height was $60 \mathrm{~cm}$ and there was a symmetrical shortening of both upper and lower limbs. Dislocation of the heads of the radii limited supination of the elbows. The hands were short and squat.

The hips were displaced; hyperlaxity of the knees was also observed.

The $\mathrm{X}$-ray features are described in the next section.
Laboratory tests and urinary mucopolysaccharides were normal; caryogram was normal, $46 \mathrm{XX}$.

The congenital displacement of the hips was treated successfully with traction and plaster casts.

After discharge the patient was not seen again until she was 19 years old. The facial features were even more evident and the scoliosis had progressed to reach more than 150 degrees; her height was $85 \mathrm{~cm}$ (Fig.1). Intelligence was normal, although she was inclined to refuse inter-personal relationship because of her physical handicaps.

Skin fibroblast cultures and lysosome hydrolases were normal.

\section{Case 2}

Third daughter of the same parents came to our observation at the age of four years. Another sister, aged five, was normal.
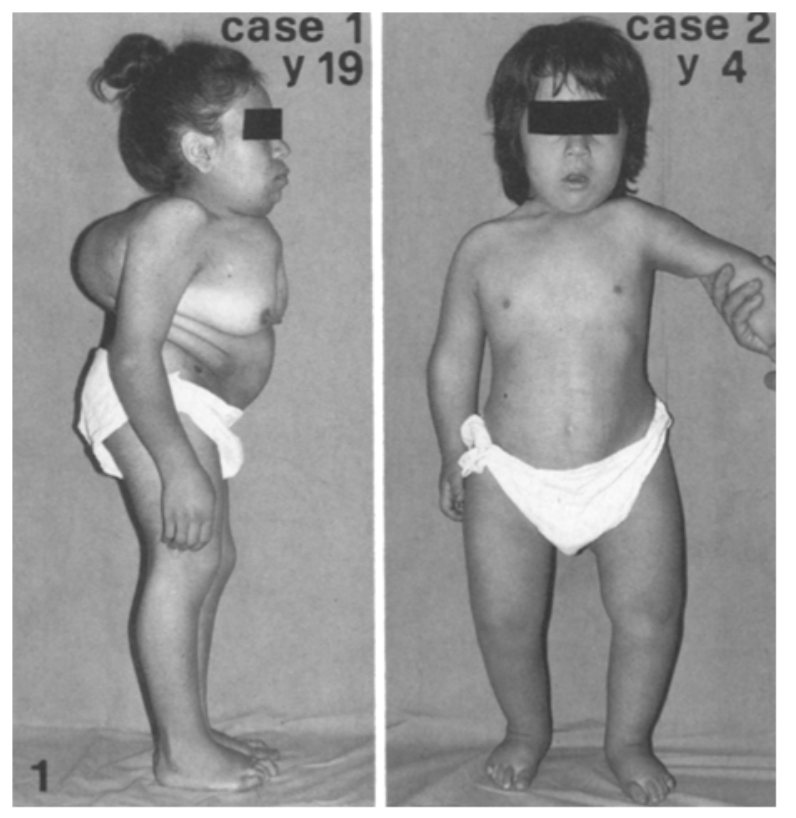

Fig. 1. Case 1, age 19: short stature and scoliosis. Case 2, age 4: short stature and features of the face 

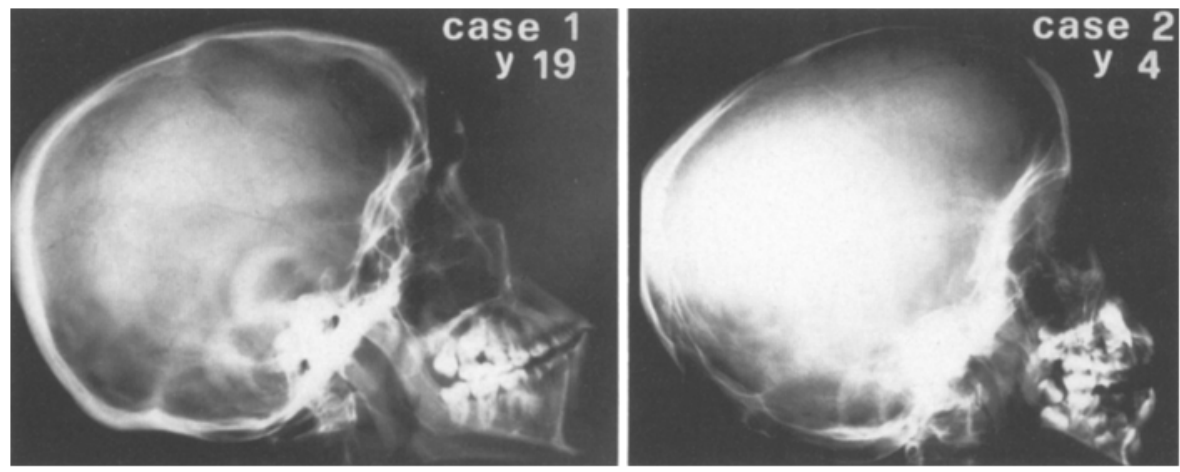

Fig.2. Abnormally wide posterior fossa of the skull; the anterior fossa is nearly vertical and sunken at the level of the chiasma. Case 1: the horizontal branch of the mandible is abnormally developed and the vertical branch is thinner than normal. Case 2: the facial bones are hypoplastic, with a small and
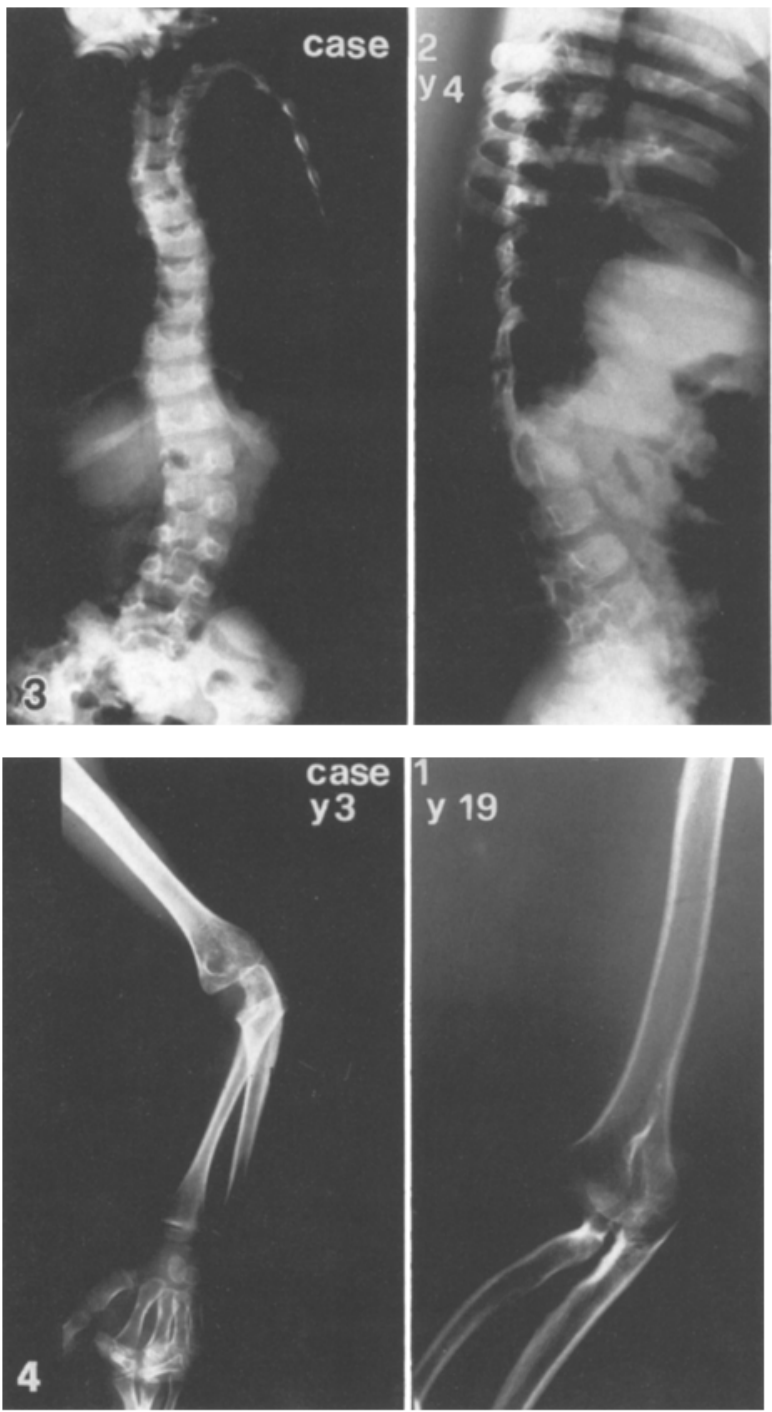

The child was $58 \mathrm{~cm}$ tall; her head was large and her face presented the same characteristics as the first sister (Fig.1). Her neck was short and scoliosis was present. The upper limbs were short with some degree of flexion and pronation of the elbows; displacement of the radial head was observed on both sides. The hands had short fingers. The hips were displaced but they could be

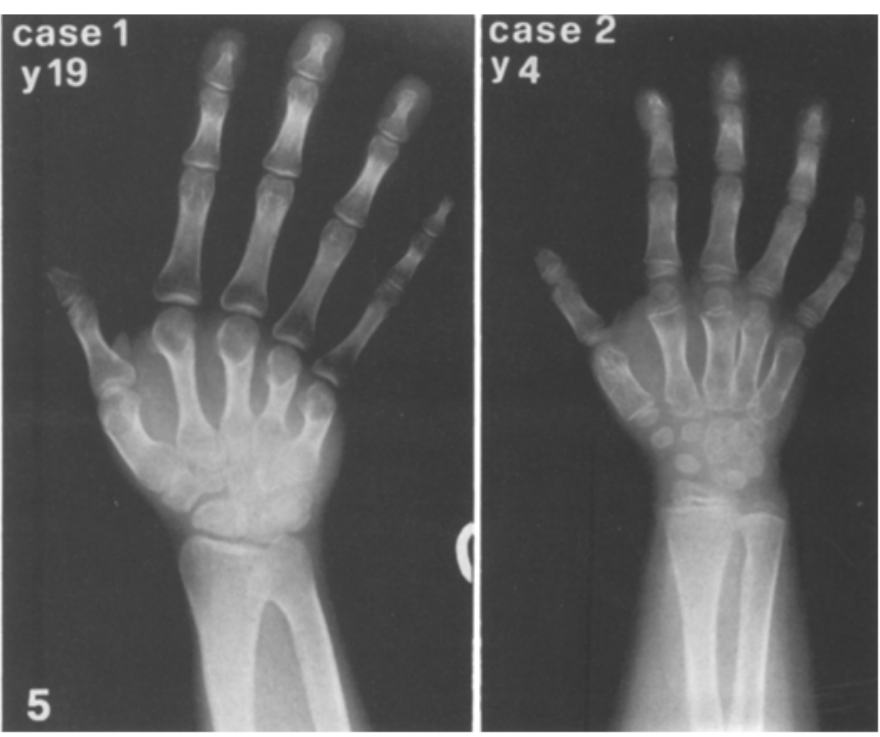

Fig. 5. Cases 1 and 2: short metacarpals and bowing of the first. In case 1 a Madelung-like deformity of the distal radius-ulnar joint is also present

Fig. 3. Case 2: mild scoliosis with four curves and kyphosis

Fig.4. Case 1 at the age of 3 and 19 years: anterior dislocation of the radial heads; the radial diaphysis is thin, while the distal metaphysis is of normal width

reduced easily with flexion and abduction. The lower limbs were short. Intelligence was normal. Cariogram was $46 \mathrm{XX}$, normal.

Laboratory tests, urinary mucopolysaccharides and hydrolases tested in serum and in cultured fibroblasts were normal. The child was kept in a divaricator for one year but no improvement of the hip laxity was obtained. 

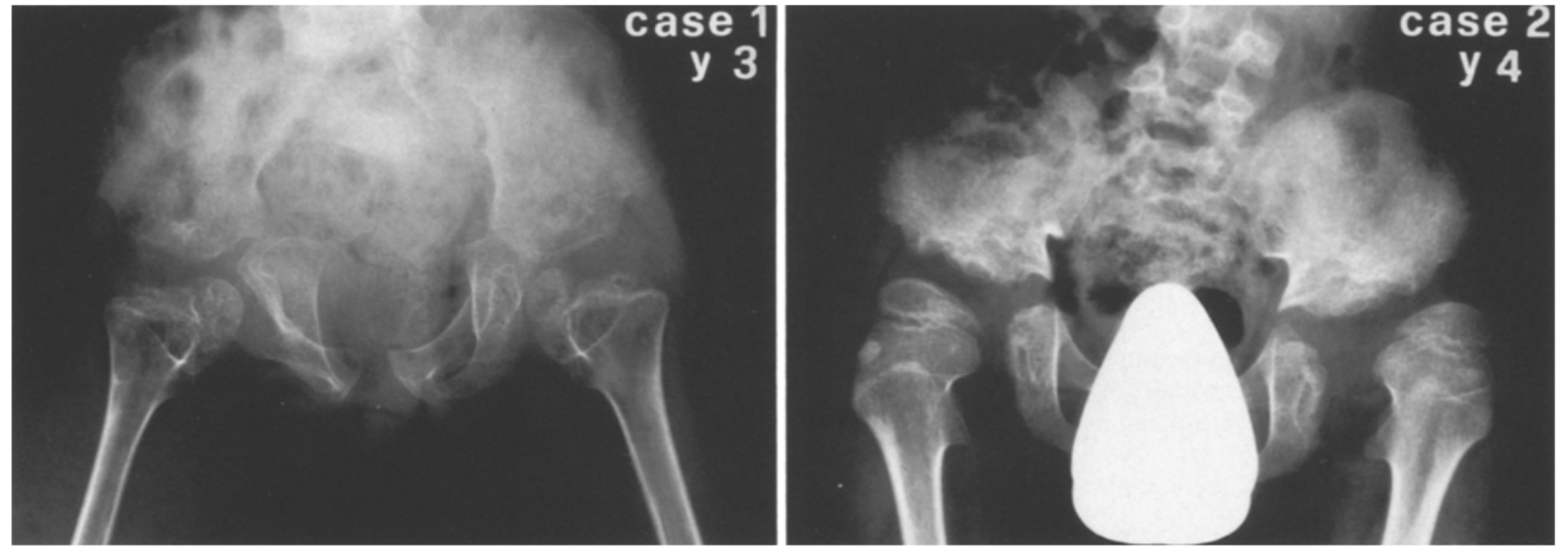

Fig. 6. Case 1 at the age of 3 years and case 2 at the age of 4 years: wide acetabula with an horizontal and irregular border; valgus of the proximal femora and irregular epiphyseal centers (hypoplastic on the medial side)
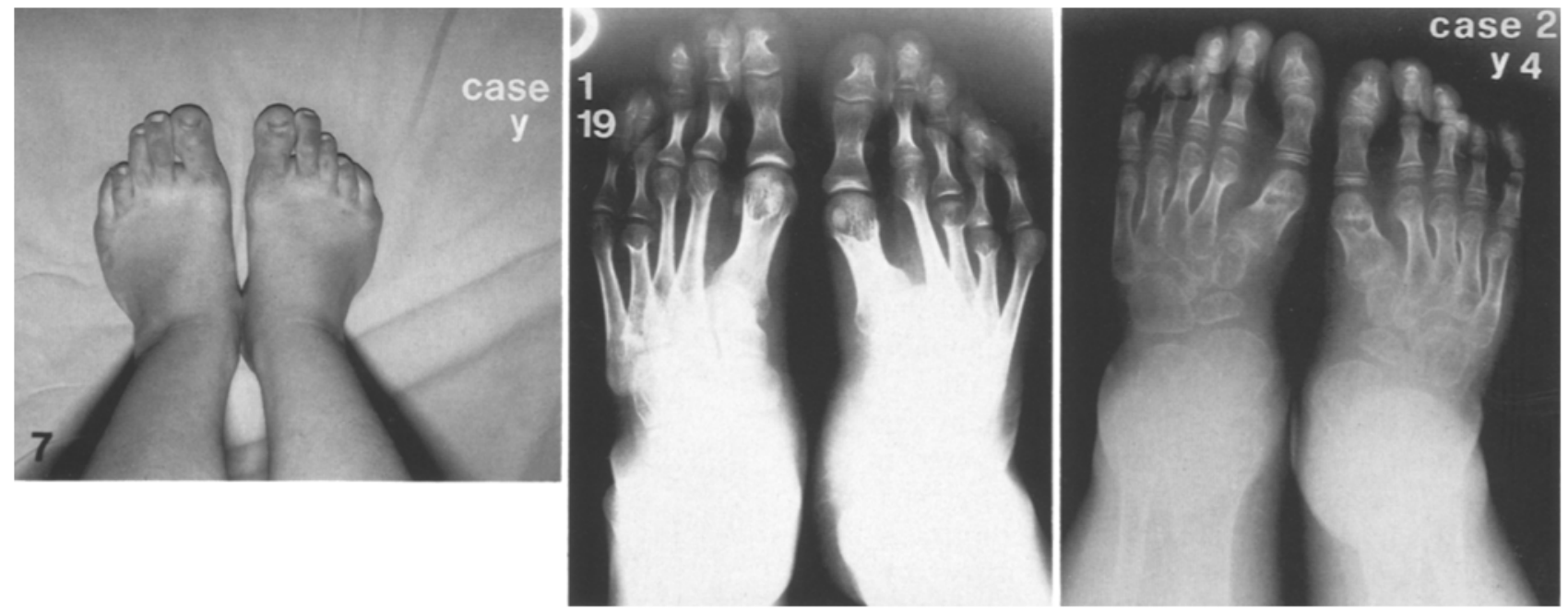

Fig. 7. Cases 1 and 2: short and squat first ray of the feet with varus first metatarsal. Case 1: irregular metatarsals with shortening of the 4th, 5th right and 3rd, 4th, 5th left. Pseudo-cystic cavities are present in the distal ends of metatarsals

\section{Radiographic features}

The skull presented in both sisters an abnormally wide posterior fossa; the anterior fossa was nearly vertical and sunken at the level of the chiasma. The parietal bones were thicker than normal and the facial bones were hypoplastic, with a small and short mandible. In the older sister, at the end of the skeletal growth, the horizontal branch of the mandible appeared abnormally developed and the vertical branch was thicker than normal (Fig.2).

The younger sister had a mild scoliosis (less than $30^{\circ}$ ) with four curves and a kyphosis with apex at the 12th dorsal and 1st lumbar vertebrae. These vertebral bodies had a hypoplastic superior edge (Fig. 3).

In the older sister a single curve scoliosis of $65^{\circ}$ was present at the age of three, and it had increased to more than $160^{\circ}$ at maturity. The deformity of the chest made difficult to evaluate the morphology of the ribs in this girl, which presented irregular calcification of the chondro-sternal cartilages; in the younger sister, on the contrary, they were abnormally thick at the lateral and anterior segments.
The upper limb bones showed a mesomelic shortening; the radius had a thin diaphysis contrasting with the normal width of the distal metaphysis and a bowed proximal end. In both sisters the radial heads were dislocated anteriorly and the distal radioulnar joints had a Madelung-like deformity (Fig. 4).

Carpal ossification centers were normal for the age. All metacarpals were short, with some shorter than the others; the first was also bowed. Proximal and middle phalanges were normal, while the distal had an enlarged base (Fig. 5).

The pelvis was anteverted. Acetabula were wide, horizontal and with an irregular border.

The proximal epiphyseal center of the femurs were hypoplastic on the medial side and in valgus. The great trochanter ossification center had an irregular shape (Fig.6).

The lower limb bones were short, with discrepancy between tibia and fibula (the latter exceeding the former by about $1 \mathrm{~cm}$ ).

The diaphysis of the femur was thin, in contrast with the normal width of distal metaphyses, which were osteopenic. In the older sister this osteopenia had produced a crushing of the tibial plates. 
Proximal femoral metaphysis presented short neck and varus deformity.

In both sisters, at the age of three and four respectively, the feet had a short and squat first ray, with varus of the 1st metatarsal. At the end of skeletal growth in the older sister many irregularities of metatarsals were observed, with a shortened first ray, but with a still more considerable shortening of the right 4 th, 5 th and left $3 \mathrm{rd}$, 4th, 5th metatarsals.

Pseudo-cystic cavities resembling those observed in multiple exostosis disease, were present at the distal ends of these shortened metatarsals.

The hallux was shorter than the other toes; the right 3rd, 4th, 5 th and left $3 \mathrm{rd}$, 4th phalanges had excess ossification nuclei. The middle phalanx of the left 5 th toe had an hour-glass like shape (Fig. 7).

The clavicles were short, the scapulae had a squared shape with hypoplastic glenoid.

\section{Discussion}

The clinical and radiographic features of these two sister are peculiar and we found remarkable difficulty in classifying them among skeletal dysplasias. The similarities of the pathological characteristics and consanguinity of parents supported the hypothesis of a genetic dysplasia.

Cases with similar features have been reported by Desbuquois et al. [2] as "Chondrodystrophic dwarfism with anarchical ossification and multiple malformations" and under other denomination by Neimann et al. [1] and by Piussan et al. [3].

The two sister reported here had many features in common with the cases already reported in the literature, namely short stature, osteoporosis, scoliosis, dysmorphic vertebral bodies, epiphyseal and metaphyseal abnormalities, hyperlaxity of the joints, dysplasia of the acetabula and dislocation of the hips. However, ulnar deviation of the hands, and early appearance of tarsal and carpal centers observed in other cases were not present. On the contrary the profile of the skull, the bowing of proximal radius with dislocation of the radial head and the Madelung-like deformity of the distal radio-carpal joint were peculiar features.

To our knowledge only nine cases of this dysplasia have been hitherto reported: three siblings described by Piussan et al. [3] had consanguineous parents. The occurrence of the disease in two other sisters with consanguineous parents further support the autosomal recessive mode of transmission.

Case no. 1 is the oldest described in the literature and also the one with the longest follow-up.

Differential diagnosis is with the Larsen syndrome.

Acknowledgements. The authors are grateful to Dr. P. Maroteaux for the useful diagnostic indication and for the references of published cases.

\section{References}

1. Neimann N, Pierson M, Manciaux M, Sapelier J (1964) A propos d'un nouveau cas de nanisme diastrophique. Arch Fr Pédiatr 21: 957

2. Desbuquois G, Grenier B, Michel J, Rossignol C (1966) Nanisme chondrodystrophique avec ossification anarchique et polymalformations chez deux soeurs. Arch Fr Pédiatr 23: 573

3. Piussan C, Maroteaux P, Castroviejo I, Risbourg B (1975) Dysplasie osseuse avec nanisme et alterations squelettiques diffuses. Six observations. Arch Fr Pédiatr 32: 541

Accepted: 6 December 1987

Prof. U.E. Pazzaglia

Clinica Ortopedica dell'Università

I.R.C.C.S. Policlinico San Matteo

Via Tarmelli 3

I-27100 Pavia

Italy

\section{Literature in pediatric radiology (continued from p.27)}

Chirurgie Pédiatrique (Paris)

Ostéome ostéoïde du col fémoral de l'enfant et l'adolescent. Longis, B. et al. (Serv. de Chirurg. Péd., CHU Dupuytren, 2, ave. Alexis-Carrel, F-87000 Limoges, France) 29, 24 (1988)

Les décollements épiphysaires spontanés chez l'enfant porteur d'une spina bifida. Michel, F. et al. (Centre Livet, Hôpital de Lyon, 8, rue de Margnolles, F-69300 Caluire, France) 29, 29 (1988)

Epiphysiolyse externe avec „caput valga“. Brichaux, J.C. et al. (Diard, F., Serv. de Rad., Hôpital des Enfants, 168 cours de l'Argonne, F-33077 Bordeaux Cedex, France) 29, 39 (1988)

Journal de Radiologie (Paris)

Empreinte ou compression trachéale latérale due au tronc artériel brachiocéphalique chez l'enfant. Ghidalia, S. et al. (Serv. de Rad., Pav. de la Mère et de l'Enfant, CHRU de Nantes, BP 1005, F-44035 Nantes, France) 69, 205 (1988)

Les calcifications laryngo-trachéo-bronchiques idiopathiques chez l'enfant. A propos de deux observations. Thollot, F. et al. (Serv. de Rad.,
Hôp. d'Enfants 5, allée du Morvan F-54500 Vandoeuvre, France) 69 , 217 (1988)

Journal of Neuroradiology (Paris)

Oligodendrogliomes thalamiques chez l'enfant: TDM et évolution clinique. Nov, A.A. et al. (Dep. of Rad., Group Health Coopérative, Central Hosp., 200 15th Ave. East, Seattle, WA 98112, USA) 15, 23 (1988)

Diagnostic tomodensitométrique dans soixante cas d'abcès cérébral de petite taille. Wu Yujin et al. (Serv. de Neurolog., Hebei Provincial Hosp., Jichang Road, Shijiazhuang, Hebei Province, People's Rep. of China) $15,77(1988)$

Pédiatrie (Lyon)

Hydropéricarde néonatal par cathéter épicutanéo-cave en silicone. Donnou-Da Lage, M.D. et al. (Guillois, B., Serv de reanimation péd. et de néonatologie, CHU morvan, F-29279 Brest Cedex, France) 43, 23 (1988)

(continued on p.64) 